\title{
ROTATIONAL MODULATION OF SATURN KILOMETRIC RADIATION, NARROWBAND EMISSION AND AURORAL HISS
}

\author{
S.-Y. Ye*, G. Fischer ${ }^{\dagger}$, W.S. Kurth*, J. D. Menietti*, and D. A. Gurnett*
}

\begin{abstract}
Despite the axisymmetry of Saturn's internal magnetic field, a variety of magnetospheric properties and radio emissions exhibit periodic modulations due to Saturn's rotation. Previous studies have shown that Saturn kilometric radiation (SKR) and auroral hiss have two different modulation rates, one associated with each hemisphere, which also vary over the time scale of a Saturn year. The narrowband emissions exhibit dual periodicities in each hemisphere. We update the modulation analysis of Saturn's radio emissions to the end of year 2016. It is shown that the northern SKR rotation slowed to around $800^{\circ}$ /day in 2016 , while the south remained around $809^{\circ}$ /day as of late 2015 (no clear rotation signal for southern SKR in 2016). When Cassini shifted to high inclination orbits at the end of 2016, a single modulation signal of the narrowband emission showed up around $800^{\circ} /$ day. A rotational modulation signal for northern auroral hiss also showed up around $800^{\circ}$ day, but there was no signal for the southern auroral hiss.
\end{abstract}

\section{Introduction}

The modulation period of radio emissions from the gas giant planets was generally thought to be fixed. Hence the longitude system adopted for Saturn was based on the period of Saturn kilometric radiation (SKR) [Desch and Kaiser, 1981]. However, observations by the Ulysses and Cassini spacecraft revealed substantial variations of the SKR period [Lecacheux et al., 1997; Galopeau and Lecacheux, 2000; Gurnett et al., 2005]. Furthermore, the radio modulation rate of Saturn has also been found to consist of two components [Kurth et al., 2008], one associated with each hemisphere [Gurnett et al., 2009a]. The dual modulation rates are also observed in Saturn narrowband emissions and auroral hiss [Gurnett et al., 2009b; Ye et al., 2010a].

Gurnett et al. [2009a] applied a tracking filter analysis to the SKR signals observed in the northern and southern hemisphere by separating the observations by latitude, and showed

\footnotetext{
* Department of Physics and Astronomy, University of Iowa, Iowa City, IA, USA

$\dagger$ Space Research Institute, Austrian Academy of Sciences, Graz, Austria
} 
a clear asymmetry in the rotational modulation period of SKR between hemispheres. It was found that the southern SKR had a period of $\sim 10.8 \mathrm{~h}$ (rotation rate of $800^{\circ} /$ day) from 2004 to 2009, whereas the northern SKR had a period of $\sim 10.6 \mathrm{~h}$ (rotation rate of $815^{\circ}$ /day) from 2006 to 2009. These two periods started to converge in 2009 and by 2010 , several months after equinox, seemed to have merged [Gurnett et al., 2010].

Fischer et al. [2015], in addition to the tracking filter analysis, used a phase tracing method [Kurth et al., 2007; 2008] to reveal the short-term variation of SKR periodicities after equinox. They showed that the north and south SKR periods crossed each other briefly after equinox and remained phase locked (same period) from spring 2010 up to mid-2012, except for the first few months of 2011. This brief separation between the northern and southern SKR periods might have been caused by the Great White Spot (GWS). The super lightning storm of Saturn could have perturbed the polar ionosphere of the planet, disrupting the phase lock between hemispheres [Fischer et al., 2014].

Ye et al. [2016] extended the modulation analysis of SKR to the end of 2015 and showed that narrowband emissions and auroral hiss are similarly modulated at variable periods in the northern and southern hemispheres. It was shown that the rotation rate of northern SKR was faster than the southern SKR from mid-2012 to autumn 2013, followed by a one year interval of similar rotation rates and phases, before northern SKR finally became slower than the southern SKR in late 2014. They also showed the reappearance of dual periodicities of $5 \mathrm{kHz}$ narrowband emissions in 2013, after a long break since equinox. The rotation rates of auroral hiss, narrowband emissions, and those of SKR were consistent with each other when they were observed at high latitudes in early 2013.

In this study, we update the modulation analysis of Saturn radio emissions to the end of 2016. We applied the Lomb-Scargle period analysis [Lomb, 1976; Scargle 1982] (also known as Least Square Spectral Analysis) to derive the rotational modulation rates for these emissions.

\section{Saturn kilometric radiation}

Similar to auroral kilometric radiation observed at Earth, SKR is emitted by unstable populations of electrons on auroral field lines via the cyclotron maser instability [Wu and Lee, 1979]. Accordingly, recent studies [Lamy 2011; Andrews et al., 2011; Lamy et al., 2013; Cowley and Provan, 2016] suggested that the SKR modulation is associated with a rotating current system, so it is fundamentally a rotating beam. However, SKR intensifies periodically when the rotating asymmetric ring current of Saturn passes through the early morning LT sector [Mitchell et al., 2009], causing enhancement of field aligned currents that drive the auroral process [Lamy et al., 2010; Kurth et al., 2011, Menietti et al., 2011]. Therefore, SKR is mainly modulated like a clock, which means that the timing of the intensification of SKR is not affected by the observer's local time [Warwick et al., 1981], as long as the morning side source is visible to the observer [Andrews et al., 2011] or the local time of the spacecraft apoapsis is not changing rapidly [Fischer et al., 2015]. So for the tracking-filter analysis of SKR (or other similar modulation analysis), there is no need to correct the phase for Cassini's local time. The modulation rate $(\omega)$ of SKR 
can be obtained by maximizing the peak-to-peak power when we fit the SKR power to a sine function of the longitude of the Sun $(\omega t)$. A detailed description of this tracking filter analysis method can be found in Gurnett et al. [2011].
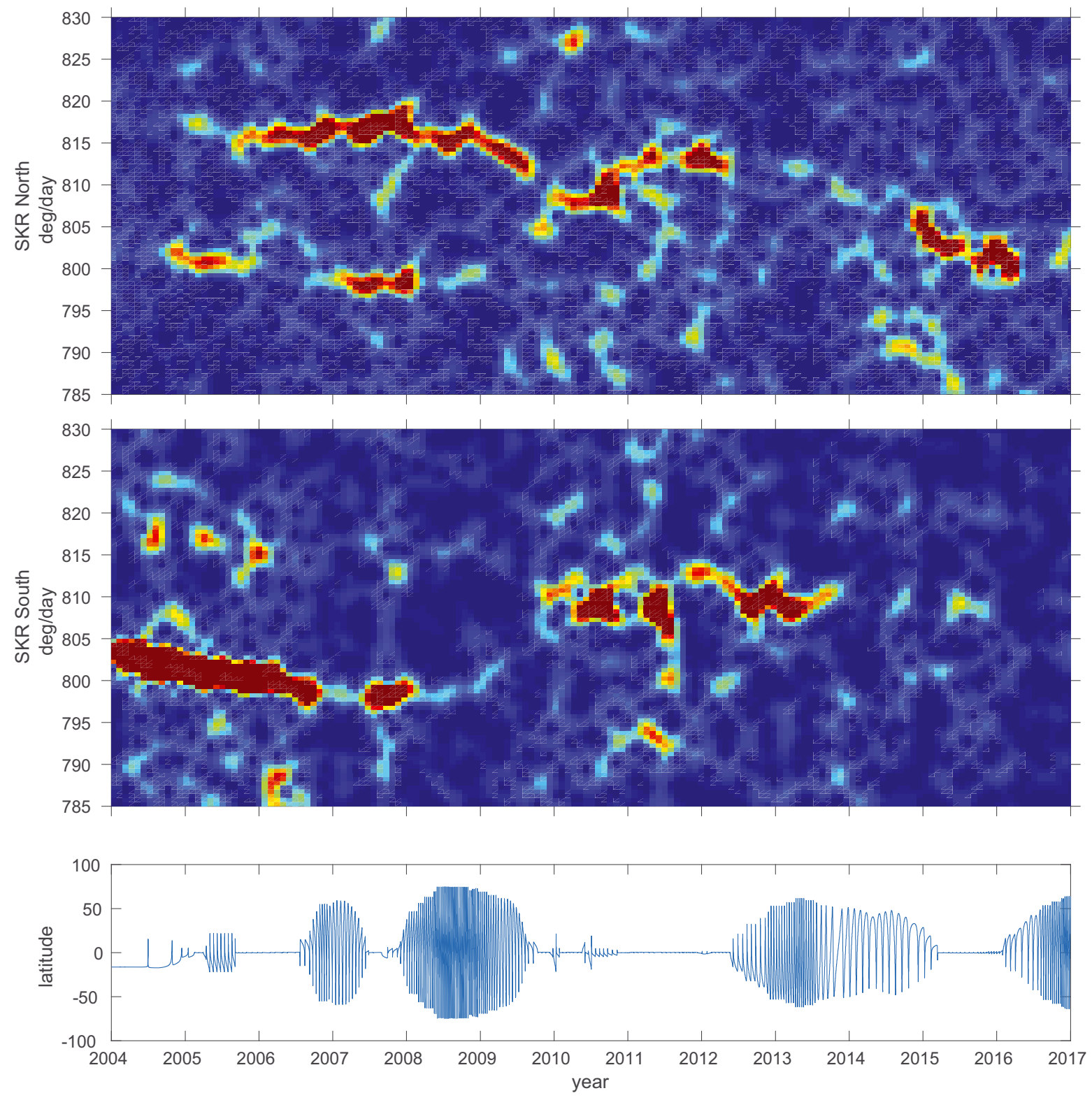

Figure 1: Lomb-Scargle modulation spectrograms of the north (top) and south (middle) SKR power (separated by polarization), observed from 2004 to 2017. The color indicates the Lomb normalized modulation power with red (100) indicating strong modulation and blue (0) indicating no modulation. The latitude of Cassini is also shown at the bottom.

Figure 1 shows Lomb-Scargle spectrograms of northern and southern SKR, which show the rotational modulation power as a function of rotation rate and time. For the northern and southern SKR powers, the wave intensities measured by the High Frequency Receiver are separated by their sense of circular polarization and integrated between 80 and $500 \mathrm{kHz}$. The integrated powers are then averaged within 10-minute windows and then normalized by the running average (65 samples, about one rotation of Saturn) to 
remove the orbital distance effect. The modulation spectral powers shown in Figure 1 are calculated by a fast Lomb algorithm [Press, 2007] from these relative intensities. The top two panels show the Lomb normalized modulation of north (top) and south (middle) SKR power. The color scale is such that red (100) indicates strong modulation and blue (0) indicates no modulation. These numbers can be converted to statistical significance levels of the modulation signals. Note that the north SKR modulation signals are sporadic from mid-2012 until late 2014 , with only three weak signals around $\sim 810^{\circ}$ /day at spring 2013, late 2013/early 2014, and autumn 2014. The south SKR modulation signals are almost absent after late 2013, except for the weak signals in mid-2014 and mid-2015. This is mainly due to the change in visibility of north/south SKR sources during Cassini's high-latitude trajectory from mid-2012 until early 2015. As shown in the bottom panel of Figure 1, before the end of 2013, Cassini spent most of its time at high southern latitudes, from where the north SKR is not visible. This gradually changed to more time spent at northern mid-latitudes where the visibility of south SKR is poor. The latest data, from the end of 2014 to the end of 2016, show that the north SKR rotation rate has decreased to $\sim 800^{\circ}$ /day, whereas the south SKR rotation rate remained at $\sim 809^{\circ} /$ day, although its rotation signal is relatively weak and not detectable in 2016. Cassini was mostly in the equatorial plane of Saturn in 2015 and shifted to high inclination orbits in 2016.

From the phase diagrams of SKR, where the wave power is plotted as a function of phase (based on an arbitrarily chosen guide rate) and time, we can determine the rotational modulation rates by tracing the phase of power maxima. The slope of the drifting phases reveals the modulation rates relative to the guide rate. Generally, a positive slope indicates a rotation rate slower than the guide rate and a negative slope indicates a rotation rate faster than the guide rate. Rotation rates determined in this way agree with our results from tracking filter or Lomb-Scargle analysis [Fischer et al., 2015; Ye et al., 2016]. The same technique can be applied to narrowband emissions and auroral hiss.

\section{Narrowband emission}

During the Voyager flyby of Saturn, a series of narrowband emissions between 3 and 30 $\mathrm{kHz}$ were detected by the Plasma Wave Science (PWS) instrument [Gurnett et al., 1981; Scarf et al., 1982]. These narrowband emissions are most prominently observed around $5 \mathrm{kHz}$ and propagate mostly in the L-O mode [Ye et al., 2009; Wang et al., 2010]. Ye et al. [2010b] showed that the L-O mode narrowband emissions are mode converted from $\mathrm{Z}$ mode narrowband emissions observed near Saturn. Less intense narrowband emissions at higher frequencies $(\sim 10-70 \mathrm{kHz})$ are found to originate from upper hybrid resonance emissions on the boundary of the plasma torus [Ye et al., 2009; Menietti et al., 2009; 2010]. A review of the studies on Saturn narrowband emissions can be found in Ye et al. [2011]. Recently, Menietti et al. [2016] surveyed possible regions of $5 \mathrm{kHz} \mathrm{Z}$ mode narrowband emissions in the lower density region of Saturn's magnetosphere and identified 6 possible source regions, one of which had relatively complete electron pitch angle coverage by the electron spectrometer onboard Cassini. Wave growth calculations based on the electron phase space distribution and cold plasma magnetoionic theory [Yoon et al., 1996 and 1998] indicated that an unstable electron population with a temperature anisotropy and 
a weak loss cone might be the source for the $5 \mathrm{kHz} \mathrm{Z}$ mode narrowband emissions.

Rotational modulation analysis of the $5 \mathrm{kHz}$ narrowband emission for the period between Saturn orbit insertion and equinox revealed two rotation rates, consistent with the northern and southern SKR [Ye et al., 2010a; Ye et al., 2016], although both modulation rates of narrowband emissions are observable in each hemisphere. This is because the original $\mathrm{Z}$ mode emissions are trapped in a region close to Saturn bounded by the upper hybrid frequency and the $\mathrm{L}=0$ cutoff frequency contour, enabling the $\mathrm{Z}$ mode to cross the equator before converting to the free space L-O mode [Ye et al., 2010b].
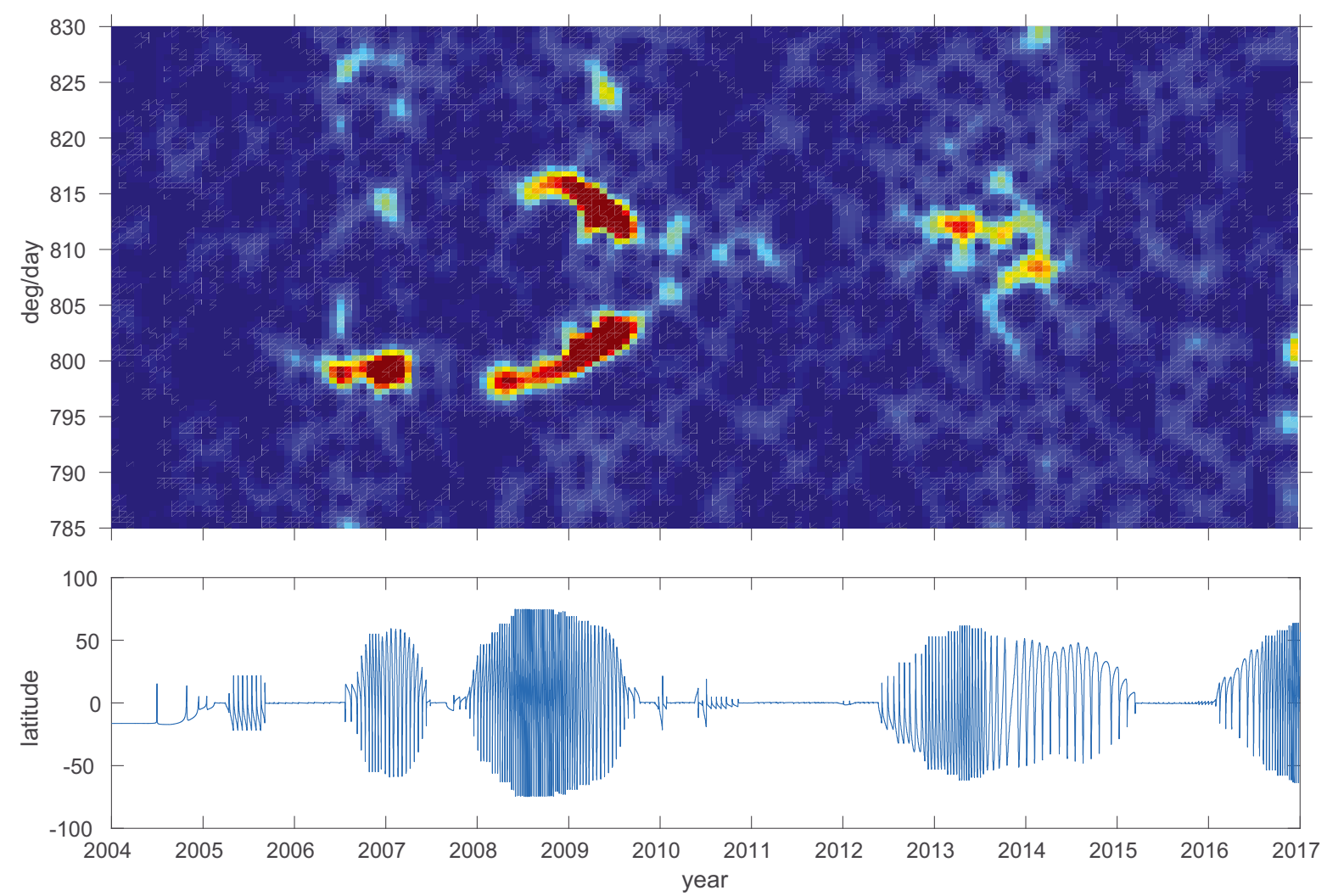

Figure 2: Lomb-Scargle modulation spectrogram of the $5 \mathrm{kHz}$ narrowband emission observed from 2004 to 2017. The color shows the Lomb normalized modulation power with red (100) indicating strong modulation and blue (0) indicating no modulation. The plot of Cassini's latitude shows that the modulation signal is preferably observed during high inclination orbits.

Figure 2 shows the Lomb-Scargle modulation spectrogram of the $5 \mathrm{kHz}$ narrowband emission from 2004 to the end of 2016. Note that the modulation signals are most intense during the high inclination orbits of Cassini (2006-2007, 2008-2009, 2013, and 2016). The dual modulation signals of narrowband emissions started to converge before equinox and seemed to merge into one weaker signal afterwards. The convergence of narrowband modulation signals into one is consistent with the SKR modulation rates analyzed by Fischer et al. [2015]. The slowing down of the narrowband modulation rate in early 2011 has been proposed to be related to the GWS [Fischer et al., 2014]. Then, after more than one year of equatorial orbits during which no modulation signal was observed for the narrowband emission, the dual modulation signals reappeared in 2013 as Cassini shifted 
to high inclination orbits and disappeared in 2014 before the orbits became equatorial. However, we can't tell which hemisphere is rotating faster in 2013 by analyzing narrowband emission data, due to the indistinguishable hemispheric origins of these emissions as discussed in Ye et al.[2010a]. At the end of 2016, a single rotational modulation signal of the narrowband emission appeared near $800^{\circ}$ /day, as Cassini shifted to high inclination orbits. The non-detection of a second rotation rate cannot be explained by the difference in observation time in two hemispheres as the signal can cross the equator before reaching the observer. This could indicate that both the northern and southern hemispheres are modulated at the same rate, or the signal from the winter hemisphere is simply too weak to be detected.

\section{Auroral hiss}

Saturn auroral hiss is a broadband whistler-mode emission commonly observed at frequencies below $100 \mathrm{~Hz}$. It shows a very pronounced rotational modulation and the rotation rates matched those of SKR in the northern and southern hemispheres of Saturn [Gurnett et al., 2009b; Ye et al., 2016]. The whistler mode auroral hiss propagates near the resonance cone at maximum growth rate and below the local plasma frequency or the local electron cyclotron frequency, whichever is lower. The ray paths form a funnel shape centered on the source field aligned current. Therefore, the spacecraft has to cut across the funnel shaped ray path of auroral hiss to detect the emission. This has three major consequences: auroral hiss can only be observed at high latitudes, the emissions appear as a rotating beam since they are confined to propagate along or within a limited angle with respect to the magnetic field, and the rotational signals associated with auroral hiss cannot cross the equator.

Since auroral hiss is generated by the downward current, which rotates with the planet, it behaves more like a rotating beam than a clock [Gurnett et al., 2009b]. Therefore, we bin the power of Saturn auroral hiss with the longitude (based on a fixed rotation period) of the spacecraft in the least square spectral analysis. Figure 3 shows the modulation spectrograms of Saturn auroral hiss in the northern and southern hemisphere. The power of auroral hiss is integrated between $2-30 \mathrm{~Hz}$ and the northern and southern components are separated based on the latitude of Cassini (e.g. latitude $>10^{\circ}$ for north auroral hiss and latitude $<-10^{\circ}$ for south auroral hiss). The modulation rates of auroral hiss exhibit a similar north-south asymmetry as SKR before mid-2009, consistent with the results of Gurnett et al. [2009b]. The modulation signals of auroral hiss after equinox are sporadic (due to limited observations at high latitudes) and often without corresponding SKR signals. Nevertheless, there is a good correspondence between southern auroral hiss and southern SKR signals in early 2013, at around $810^{\circ}$ /day. In 2016, a strong modulation signal showed up for the northern auroral hiss near $800^{\circ}$ /day as Cassini started to sample higher latitudes. There is a corresponding northern SKR signal at the same time. However, no modulation signal for the southern auroral hiss was observed. The rotational modulation analysis of Saturn auroral hiss confirmed a fundamental north-south asymmetry in the auroral phenomena at Saturn and the northern (summer) hemisphere auroral currents started to dominate after the recent equinox. 

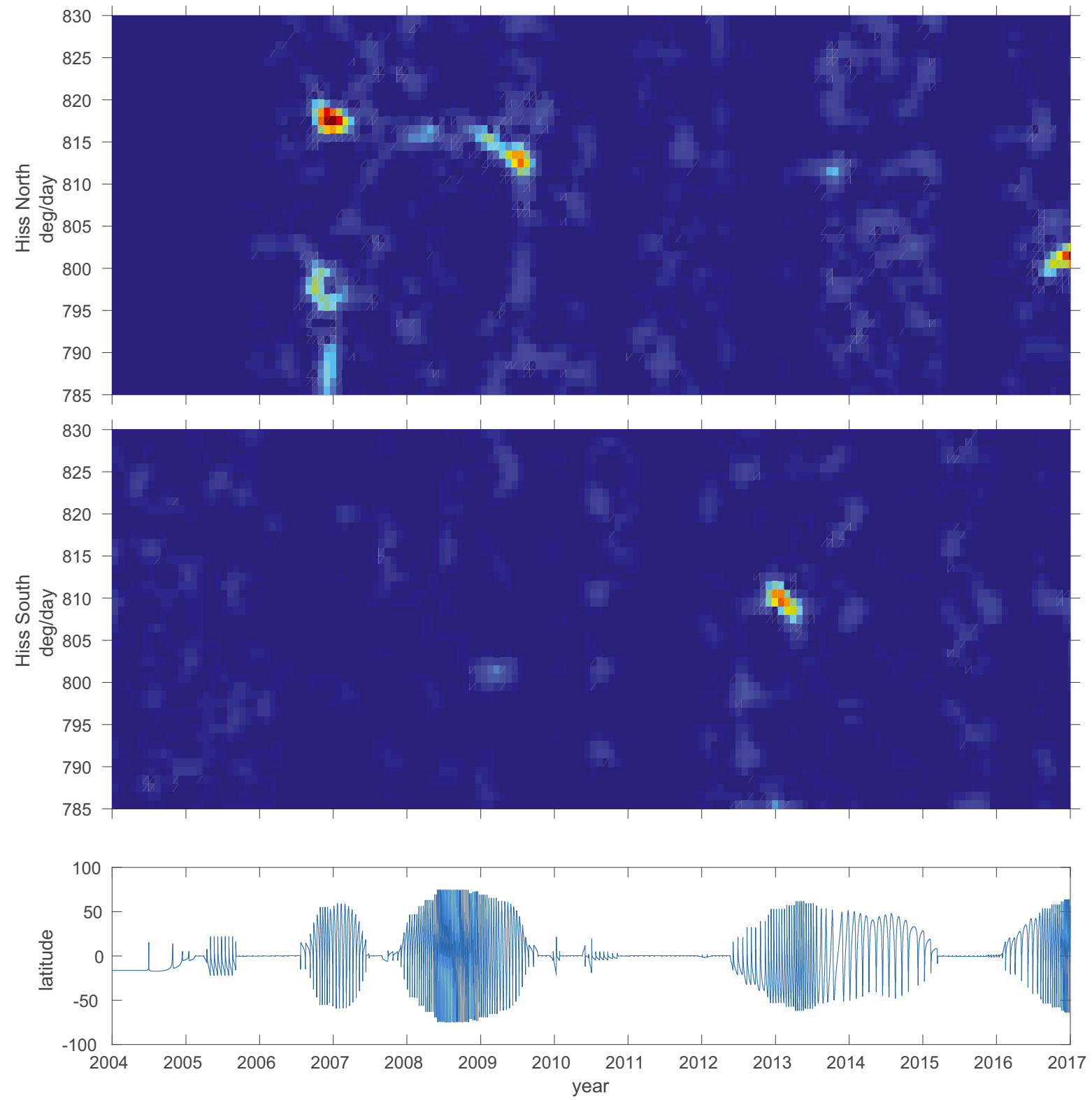

Figure 3: Least square spectral analysis of the north and south auroral hiss power, separated by latitude of observation, from 2004 to 2017. The auroral hiss powers were sorted into bins of longitude of the spacecraft before the least square fits. The color shows the square of the peak-to-peak amplitude of the fitted wave power modulation, with red (0.1) indicating strong modulation and blue (0) indicating no modulation.

\section{Discussion}

The source of the $\sim 10.7$ hour periods observed in Saturn's magnetosphere is still a mystery. Various models have been proposed to explain the observed periodicities despite the axisymmetry of the planet's internal magnetic field, which can be classified into ionosphere driven [Gurnett et al., 2009; Jia et al., 2012] and magnetosphere driven models [Gurnett et al., 2007; Goldreich and Farmer, 2007]. The ionosphere driven models have 
been supported by observations of field aligned currents that couple the polar ionosphere to the magnetosphere [Southwood and Cowley, 2014; Kivelson and Jia, 2014; Hunt et al., 2014 and 2015], although Smith et al. [2014] pointed out that the field aligned currents should instead be driven by a twin vortex circulation in the atmosphere.

Ye et al. [2016] showed that SKR, narrowband emission and auroral hiss are modulated at similar rates, which are variable on the time scale of a Saturn year. Similar variation of modulation periods have been observed in magnetic field perturbation and high energy electrons [Carbary et al., 2017 and references therein]. The observed variation of 1\% in the modulation periods is too large to arise from the deep interior due to the massive moment of inertia of Saturn [Gurnett et al., 2007]. The variable periods of radio emissions [Galopeau and Lecacheux, 2000; Gurnett et al., 2005; Fischer et al., 2015] and magnetic field oscillations [Andrews et al., 2008; 2011; 2012] indicate that the magnetic field and field-aligned currents are slipping over the neutral atmosphere in the polar region. The long-term variations in modulation periods are linked to seasonal changes in the polar regions, as the rotational modulation of the radio emissions and magnetic field perturbations also display a hemispheric asymmetry that reverses after each equinox [Gurnett et al., 2009a; Provan et al., 2016; Ye et al., 2016]. The strength of the field-aligned current is determined by the ionospheric conductivity, which changes seasonally with the solar EUV ionization in each polar ionosphere as the Sun elevation angle in the polar region changes over a Saturn year. The seasonally variable neutral atmospheric winds in the auroral zone could influence the slippage of the magnetosphere over the neutral atmosphere [Smith, 2006].

SKR is generated on auroral field lines in the upward current region whereas auroral hiss is generated by electrons moving up an auroral field line in the downward current region [Gurnett et al., 2009a and b; Schippers et al., 2011]. The upward and downward current regions should be separated by roughly $180^{\circ}$ in planetary rotation phase [Andrews et al., 2012]. Ye et al. [2016] showed that the phase difference between SKR and auroral hiss is local time dependent, because SKR is modulated like a clock (strongest source on the early morning sector) whereas auroral hiss is a rotating beam [Gurnett et al., 2009b]. It has been shown that Saturn narrowband emission is a clock-like emission [Wang et al., 2010], and it lags SKR by about $90^{\circ}$ in terms of the longitude of the Sun [Ye et al., 2010a]. The phase relations between these emissions have been compared for two periods when Cassini was at midnight and dawn local time sectors, respectively [Ye et al., 2016]. It was shown that SKR and auroral hiss are almost in anti-phase in the pre-dawn local time sector. This is consistent with the phase difference between the upward and downward currents that would be observed by a spacecraft in the dawn-side field aligned current region.

The intensity of auroral hiss was also shown to be dependent on local time [Ye et al., 2016]. The observed intensities of auroral hiss are higher at the dawn and dusk sectors. The local time preference of the average power of auroral hiss might indicate that auroral hiss is more like a flashing light, which intensifies at certain local time sectors during each rotation. The Saturn auroral hiss may only appear to be a searchlight due to its narrow emission cone. The intensification of auroral hiss on the dawn and dusk sides is consistent with the rotating field aligned currents intensifying on the dawn side due 
to the interaction of Saturn's magnetic field with the solar wind, which leads to Dungey type reconnections at high latitudes [Southwood and Chané, 2016]. The dawn side upward current, responsible for the generation of SKR, draws a downward current on the opposite side of the planet through the polar ionosphere, which leads to intensification of auroral hiss on the dusk side.

\section{Summary}

We extended the rotational modulation analysis of SKR, auroral hiss and narrowband emission to the end of 2016. The Lomb-Scargle analysis of polarization-separated SKR power time series showed that the northern SKR rotation slowed to $\sim 800^{\circ}$ /day in 2016 whereas the southern SKR rotation remained at $\sim 809^{\circ}$ day in 2015 . There is no clear southern SKR modulation signal in 2016. The dual rotational modulation rates of narrowband emission (hemispherical origins not distinguishable) reappeared in 2013, consistent with SKR modulation rates in the same period. In 2016, Cassini shifted to high inclination orbits, favoring the observation of narrowband emissions. Rather than the dual periods observed during the previous high inclination orbit intervals, a single rotational modulation signal appeared near $800^{\circ}$ / day at the end of 2016 . The hemispherical origins of auroral hiss are distinguishable by the latitude of observation. Least square spectral analysis applied to auroral hiss power time series showed distinct rotational modulation rates in the northern and southern hemispheres, consistent with SKR modulation rates before mid-2013, but slightly different afterwards. However, auroral hiss and narrowband emission showed agreement in modulation rates in 2013 despite the ambiguity in the hemispherical origin of narrowband emissions. Towards the end of 2016, a strong modulation signal appeared for the northern auroral hiss near $800^{\circ}$ /day but no modulation signal for the southern auroral hiss was observed. The field aligned currents responsible for generating auroral hiss rotate with the planet and intensify on the dawn and dusk sides, which is consistent with the observation that the phase difference between SKR and auroral hiss as well as the intensity of auroral hiss are local time dependent. These results confirm that the variation of rotational periodic modulations in Saturn's magnetosphere is seasonal, as the rotational modulation has shifted from southern dominance before equinox to northern dominance afterwards. The local time dependence of SKR and auroral hiss is consistent with the transient enhancement of field aligned currents on the morning side due to the Dungey type reconnection [Southwood and Chané, 2016], which might be the source of the clock signal. Hunt et al. [2016] found the rotating field aligned currents to be slightly stronger at dawn-noon sector than at midnight, but this enhancement is not enough to account for the local time asymmetry in SKR power. Cassini probably didn't pass through the morning side auroral field lines during the time of reconnection to observe the most enhanced field aligned currents, while continuous radio observation can detect the most intense SKR anytime from a distance.

Acknowledgments. This paper benefited from the discussions in the international team workshop "Rotational phenomena in Saturn's magnetosphere" funded by the International Space Science Institute (ISSI) in Bern, Switzerland. The Editors thank Ulrich Taubenschuss and one anonymous reviewer for their help in evaluating this paper. 


\section{References}

Andrews, D. J., E. J. Bunce, S. W. H. Cowley, M. K. Dougherty, G. Provan, and D. J. Southwood, Planetary period oscillations in Saturn's magnetosphere: Phase relation of equatorial magnetic field oscillations and Saturn kilometric radiation modulation, J. Geophys. Res., 113, A09205, 2008.

Andrews, D. J., B. Cecconi, S. W.H. Cowley, M. K. Dougherty, L. Lamy, G. Provan, and P. Zarka, Planetary period oscillations in Saturn's magnetosphere: Evidence in magnetic field phase data for rotational modulation of Saturn kilometric radiation emissions, J. Geophys. Res., 116, A09206, 2011.

Andrews, D. J., S. W. H. Cowley, M. K. Dougherty, L. Lamy, G. Provan, and D. J. Southwood, Planetary period oscillations in Saturn's magnetosphere: Evolution of magnetic oscillation properties from southern summer to post-equinox, J. Geophys. Res., 117, A04224, 2012.

Carbary, J. F., Update on Saturn's energetic electron periodicities, J. Geophys. Res., 122, 156-165, 2017.

Cowley, S. W. H. and G. Provan, Planetary period oscillations in Saturn's magnetosphere: Further comments on the relation between post-equinox properties deduced from magnetic field and Saturn kilometric radiation measurements, Icarus, 272, 258-276, 2016.

Desch, M. D., and M. L. Kaiser, Voyager measurement of the rotation period of Saturn's magnetic field, Geophys. Res. Lett., 8, 253-256, 1981.

Fischer, G., S.-Y. Ye, J. B. Groene, A. P. Ingersoll, K. M. Sayanagi, J. D. Menietti, W. S. Kurth, and D. A. Gurnett, A possible influence of the Great White Spot on Saturn kilometric radiation periodicity, Ann. Geophys., 32, 1463-1476, 2014.

Fischer, G., D. A. Gurnett, W.S. Kurth, S.-Y. Ye, and J. B. Groene, Saturn kilometric radiation periodicity after equinox, Icarus, 254, 72-91, 2015.

Galopeau, P. H. M., and A. Lecacheux, Variations in Saturn's radio rotation period measured at kilometer wavelengths, J. Geophys. Res., 105, 13089-13101, 2000.

Gurnett, D. A., W.S. Kurth, and F. L. Scarf, Narrowband electromagnetic emissions from Saturn's magnetosphere, Nature, 292, 733-737, 1981.

Gurnett, D. A., et al. (26 co-authors), Radio and plasma wave observations at Saturn from Cassini's approach and first orbit, Science, 307, 1255-1259, 2005.

Gurnett, D. A., A. M. Persoon, W. S. Kurth, J. B. Groene, T. F. Averkamp, M. K. Dougherty, and D. J. Southwood, The variable rotation period of the inner region of Saturn's plasma disk, Science, 316, 442-445, doi:10.1126/science.1138562, 2007.

Gurnett, D. A., A. Lecacheux, W. S. Kurth, A. M. Persoon, J. B. Groene, L. Lamy, P. Zarka, and J. F. Carbary, Discovery of a north-south asymmetry in Saturn's radio rotation period, Geophys. Res. Lett., 36, L16102, 2009a. 
Gurnett, D. A., A. M. Persoon, J. B. Groene, A. J. Kopf, G. B. Hospodarsky, and W. S. Kurth, A north-south difference in the rotation rate of auroral hiss at Saturn: Comparison to Saturn's kilometric radio emission, Geophys. Res. Lett., 36, L21108, $2009 \mathrm{~b}$

Gurnett, D. A., J. B. Groene, A. M. Persoon, J.D. Menietti, S.-Y. Ye, W.S. Kurth, R. J. MacDowall, and A. Lecacheux, The reversal of the north and south modulation rates of the north and south components of Saturn kilometric radiation near equinox, Geophys. Res. Lett., 37, L24101, doi:1029/2010GL045796, 2010.

Gurnett, D. A., J. B. Groene, T.F. Averkamp, W.S. Kurth, S.-Y. Ye, and G. Fischer, An SLS4 longitude system based on a tracking filter analysis of the rotational modulation of Saturn kilometric radiation, in Planetary Radio Emissions VII, edited by H. O. Rucker, W. S. Kurth, P. Louarn, and G. Fischer, Austrian Academy of Sciences Press, Vienna, 51-64, 2011.

Hunt, G. J., S. W.H. Cowley, G. Provan, E. J. Bunce, I. I. Alexeev, E. S. Belenkaya, V. V. Kalegaev, M.K. Dougherty, and A. J. Coates, Field-aligned currents in Saturn's southern nightside magnetosphere: Subcorotation and planetary period oscillation components, J. Geophys. Res., 119, 9847-9899, 2014.

Hunt, G. J., S.W.H. Cowley, G. Provan, E. J. Bunce, I. I. Alexeev, E. S. Belenkaya, V. V. Kalegaev, M. K. Dougherty, and A. J. Coates, Field-aligned currents in Saturn's northern nightside magnetosphere: Evidence for interhemispheric current flow associated with planetary period oscillations, J. Geophys. Res., 120, 7552-7584, 2015 .

Hunt, G. J., S.W.H. Cowley, G. Provan, E. J. Bunce, I. I. Alexeev, E. S. Belenkaya, V. V. Kalegaev, M. K. Dougherty, and A. J. Coates, Field-aligned currents in Saturn's magnetosphere: Local time dependence of southern summer currents in the dawn sector between midnight and noon, J. Geophys. Res., 121, 7785-7804, 2016.

Kivelson, M. G., and X. Jia, Control of periodic variations in Saturn's magnetosphere by compressional waves, J. Geophys. Res., 119, 8030-8045, 2014.

Kurth, W.S., A. Lecacheux, T. F. Averkamp, J. B. Groene, and D. A. Gurnett, A Saturn longitude system based on a variable kilometric radiation period, Geophys. Res. Lett., 34, L02201, 2007.

Kurth, W. S., T. F. Averkamp, D. A. Gurnett, J. B. Groene, and A. Lecacheux, An update to a Saturnian longitude system based on kilometric radio emissions, J. Geophys. Res., 113, A05222, 2008.

Kurth, W.S., et al. (20 co-authors), A close encounter with a Saturn kilometric radiation source region, in Planetary Radio Emissions VII, edited by H. O. Rucker, W.S. Kurth, P. Louarn, and G. Fischer, Austrian Academy of Sciences Press, Vienna, 75-85, 2011.

Lamy, L., Variability of southern and northern SKR periodicities, in Planetary Radio Emissions VII, edited by H. O. Rucker, W.S. Kurth, P. Louarn, and G. Fischer, Austrian Academy of Sciences Press, Vienna, 39-50, 2011. 
Lamy, L., P. Schippers, P. Zarka, B. Cecconi, C. S. Arridge, M. K. Dougherty, P. Louarn, N. André, W.S. Kurth, R. L. Mutel, D. A. Gurnett, and A. J. Coates, Properties of Saturn kilometric radiation measured within its source region, Geophys. Res. Lett., 37, L12104, doi:10.1029/2010GL043415, 2010.

Lamy, L., R. Prangé, W. Pryor, J. Gustin, S. V. Badman, H. Melin, T. Stallard, D. G. Mitchell, and P. C. Brandt, Multispectral simultaneous diagnosis of Saturn's aurorae throughout a planetary rotation, J. Geophys. Res., 118, 4817-4843, 2013.

Lecacheux, A., P. Galopeau, and M. Aubier, Re-visiting Saturnian kilometric radiation with Ulysses/URAP, in Planetary Radio Emissions IV, edited by H. O. Rucker, S. J. Bauer, and A. Lecacheux, Austrian Academy of Sciences Press, Vienna, 313325, 1997.

Lomb, N. R., Least-squares frequency analysis of unequally spaced data, Astrophys. Space Sci., 39, 447-462, 1976.

Menietti, J. D., S.-Y. Ye, P.H. Yoon, O. Santolik, A. M. Rymer, D. A. Gurnett, and A. J. Coates, Analysis of narrowband emission observed in the Saturn magnetosphere, J. Geophys. Res., 114, A06206, 2009.

Menietti, J. D., P. H. Yoon, S.-Y. Ye, B. Cecconi, and A. M. Rymer, Source mechanism of Saturn narrowband emission, Ann. Geophys., 28, 1013-1021, 2010.

Menietti, J. D., R. L. Mutel, P. Schippers, S.-Y. Ye, D. A. Gurnett, and L. Lamy, Analysis of Saturn kilometric radiation near a source center, J. Geophys. Res., 116, A12222, 2011.

Menietti, J. D., P. D. Yoon, D. Pisa, S.-Y. Ye, O. Santolik, C. S. Arridge, D. A. Gurnett, and A. J. Coates, Source region and growth analysis of narrowband Z-mode emission at Saturn, J. Geophys. Res., 121, 11929-11942, 2016.

Mitchell, D. G., S. M. Krimigis, C. Paranicas, P. C. Brandt, J. F. Carbary, E. C. Roelof, W.S. Kurth, D. A. Gurnett, J. T. Clarke, J. D. Nichols, J.-C. Gérard, D. C. Grodent, M.K. Dougherty, and W.R. Pryor, Recurrent energization of plasma in the midnight-to-dawn quadrant of Saturn's magnetosphere, and its relationship to auroral UV and radio emissions, Planet. Space Sci., 57, 1732-1742, 2009.

Press, W.H., Numerical recipes 3rd edition: The art of scientific computing, Cambridge University Press, Cambridge, UK, 2007.

Provan, G., S. W. H. Cowley, L. Lamy, E. J. Bunce, G. J. Hunt, P. Zarka, and M. K. Dougherty, Planetary period oscillations in Saturn's magnetosphere: Coalescence and reversal of northern and southern periods in late northern spring, J. Geophys. Res., 121, 9829-9862, 2016.

Scarf, F. L., D. A. Gurnett, W.S. Kurth, and R. L. Poynter, Voyager-2 plasma wave observations at Saturn, Science, 215, 587-594, 1982.

Scargle, J. D., Studies in astronomical time series analysis. II. Statistical aspects of spectral analysis of unevenly spaced data, Astrophys. J., 263, 835-853, 1982. 
Schippers, P., C. S. Arridge, J. D. Menietti, D. A. Gurnett, L. Lamy, B. Cecconi, D. G. Mitchell, N. André, W. S. Kurth, S. Grimald, and M. K. Dougherty, Auroral electron distributions within and close to the Saturn kilometric radiation source region, $J$. Geophys. Res, 116, A05203, 2011.

Smith, C. G. A., Periodic modulation of gas giant magnetospheres by the neutral upper atmosphere, Ann. Geophys., 24, No. 10, 2709-2717, 2006.

Southwood, D. J., and E. Chané, High-latitude circulation in giant planet magnetospheres, J. Geophys. Res., 121, 5394-5403, 2016.

Southwood, D. J., and S.W.H. Cowley, The origin of Saturn's magnetic periodicities: Northern and southern current systems, J. Geophys. Res., 119, 1563-1571, 2014.

Wang, Z., D. A. Gurnett, G. Fischer, S.-Y. Ye, W.S. Kurth, D. G. Mitchell, J. S. Leisner, and C. T. Russell, Cassini observations of narrowband radio emissions in Saturn's magnetosphere, J. Geophys. Res., 115, A06213, 2010.

Warwick, J. W., J. B. Pearce, D. R. Evans, T. D. Carr, J. J. Schauble, J. K. Alexander, M. L. Kaiser, M. D. Desch, B. M. Pedersen, A. Lecacheux, G. Daigne, A. Boischot, and C.H. Barrow, Planetary Radio Astronomy observations from Voyager 1 near Saturn, Science, 212, 239-243, 1981.

Wu, C. S., and L. C. Lee, A theory of terrestrial kilometric radiation, Astrophys. J., 230, 621-626, 1979.

Ye, S.-Y., D. A. Gurnett, G. Fischer, B. Cecconi, J. D. Menietti, W. S. Kurth, Z. Wang, G. B. Hospodarsky, P. Zarka, and A. Lecacheux, Source locations of narrowband radio emissions detected at Saturn, J. Geophys. Res., 114, A06219, 2009.

Ye, S.-Y., D. A. Gurnett, J. B. Groene, Z. Wang, and W. S. Kurth, Dual periodicities in the rotational modulation of Saturn narrowband emissions, J. Geophys. Res., 115, A12258, 2010a.

Ye, S.-Y., J. D. Menietti, G. Fischer, Z. Wang, B. Cecconi, D. A. Gurnett, and W. S. Kurth, $\mathrm{Z}$ mode waves as the source of Saturn narrowband radio emissions, J. Geophys. Res., 115, A08228, 2010b.

Ye, S.-Y., G. Fischer, J.D. Menietti, Z. Wang, D. A. Gurnett, and W.S. Kurth, An overview of Saturn narrowband radio emissions observed by Cassini RPWS, in Planetary Radio Emissions VII, edited by H. O. Rucker, W. S. Kurth, P. Louarn, and G. Fischer, Austrian Academy of Sciences Press, Vienna, 99-113, 2011.

Ye, S.-Y., G. Fischer, W. S. Kurth, J. D. Menietti, and D. A. Gurnett, Rotational modulation of Saturn's radio emissions after equinox, J. Geophys. Res., 121, 11714-11728, 2016.

Yoon, P.H., A.T. Weatherwax, T. J. Rosenberg, and J. LaBelle, Lower ionospheric cyclotron maser theory: A possible source of $2 f_{c e}$ and $3 f_{c e}$ auroral radio emissions, $J$. Geophys. Res., 101, A12, 27015-27026, 1996. 
Yoon, P. H., A. T. Weatherwax, and T. J. Rosenberg, On the generation of auroral radio emissions at harmonics of the lower ionospheric electron cyclotron frequency: $\mathrm{X}, \mathrm{O}$ and Z mode maser calculations, J. Geophys. Res., 103, A3, 4071-4078, 1998. 